\title{
Review on Methods of test gear transmissions
}

\author{
Jorge L. Moya Rodríguez ${ }^{1}$, Guillermo Abreu Ruano², Rafael Goytisolo Espinosa ${ }^{3}$, Maida Bárbara Reyes Rodríguez ${ }^{4}$ \\ ${ }^{1}$ ITEGAM. Brasil. Email: jorgemoyar@gmail.com \\ ${ }^{2}$ Electroquímica de Sagua la Grande. Cuba. Email: guilleabreuruano@gmail.com \\ ${ }^{3}$ Universidad "Carlos Rafael Rodríguez" de Cienfuegos Email: ragoyti@ucf.edu.cu \\ ${ }^{4}$ Facultad de Ingeniería Mecánica. Universidad Central "Marta Abreu” de Las Villas. Email: maidab@uclv.edu.cu
}

\begin{abstract}
The test beds for gear transmissions tests, enable the simulation of real contact conditions, generated on the gear teeth mesh and its behaviour evaluation, under established conditions, reliably fixed and monitored, such as: speed, torque, lubricant temperature, and others. These tests are used for lubrication analysis, wear and major destructive phenomena with negative influence on the gears life. This paper present the design of two test rigs for spur gears assessment, focusing the Contact Fatigue Strength and bending tests, which are the two main design criteria applying to the gears transmissions. These machines allow the behavior evaluation about such phenomena of different materials types, lubricants, additives, geometry modification, and other issues affecting the gears performance. It also establishes a test procedure, and just provides all planes for the machine manufacture and test specimens.
\end{abstract}

Keywords: Gears, Failure prediction, Gear test, Contact fatigue, Bending fatigue.

\section{Review sobre Métodos de Ensayo de transmisiones por engranajes}

\section{RESUMEN}

Los bancos de prueba para el ensayo de transmisiones por engranajes, permiten simular las condiciones del contacto real que se genera entre los dientes de estos elementos, para evaluar su comportamiento ante determinadas variables que pueden ser establecidas y controladas, por ejemplo: velocidad de rotación, carga aplicada, temperatura del lubricante, entre otras. Estos ensayos son utilizados para el estudio de la lubricación, el desgaste y los principales fenómenos destructivos que inciden negativamente sobre la vida útil de los engranajes. En este trabajo se presenta el diseño de dos bancos de prueba destinados a la evaluación de engranajes cilíndricos de dientes rectos, concebidos para realizar ensayos de resistencia a la fatiga por contacto y por flexión, que son precisamente los dos principales criterios de diseño aplicados a las transmisiones por engranajes. Mediante el uso de estas máquinas es posible evaluar el comportamiento ante estos fenómenos, de diferentes tipos de materiales, lubricantes, aditivos, cambios de geometría, y otras cuestiones que inciden en el desempeño de los engranajes. Se establece además una metodología para la conducción de los ensayos, y se adjuntan todos los planos necesarios, para la construcción de las máquinas y los especimenes de ensayo.

Palabras clave: Engranajes, Predicción de fallas, Ensayo de engranajes, Fatiga por contacto, Fatiga por flexión.

\section{INTRODUCCIÓN}

Las fallas inesperadas en los elementos de máquinas, durante su explotación, pueden desencadenar en situaciones catastróficas, tales como la pérdida de equipos, o lo que resulta peor aún, de la vida de seres humanos. Los engranajes son componentes mecánicos utilizados en las transmisiones de potencia y frecuentemente son responsables de averías que colapsan el normal funcionamiento de estos sistemas. Por esta razón, el análisis y predicción de las fallas en engranajes, ha recibido una considerable atención y se han creado alrededor del mundo, varios centros e instituciones dedicados a su estudio, con el objetivo de prevenirlas y evitar, con un alto grado de confianza, los efectos indeseados que de ellas se derivan.

Los engranajes son utilizados para transmitir fuerza y movimiento, en una amplia variedad de aplicaciones, donde suelen desempeñar su trabajo, sometidos a la más diversa gama de condiciones y regímenes de explotación, en ocasiones 
bastante severos, por lo que resulta de gran utilidad poder contar con bancos de ensayo, que permitan prever el comportamiento esperado de estos elementos, ante diferentes circunstancias.

Estas instalaciones posibilitan la realización de ensayos acelerados de vida a la fatiga y permiten:

- Evaluar la capacidad de carga portante de engranajes y lubricantes, ante diferentes modos de fallas.

- Explorar e investigar los limites de endurancia o resistencia, de engranajes sometidos a tensiones de contacto y tensiones de flexión, con diferentes combinaciones de materiales y tratamientos térmicos.

- Investigar la influencia que provocan sobre los fenómenos de fallas, aspectos tales como: tipo y viscosidad del lubricante, aditivos, correcciones en la geometría del diente, entre otros.

- Determinar la eficiencia en transmisiones por engranajes con diferentes geometrías o perfiles del diente.

- Elaborar bases de datos, que sirvan de soporte al desarrollo de programas, para optimizar los procesos de diseño, análisis y elaboración de engranajes.

- Perfeccionar el diseño de materiales y la vida de componentes, mediante un mejor conocimiento de los mecanismos de fatiga.

- Mejorar la seguridad, fiabilidad y vida útil de las transmisiones por engranajes.

- Reducir el peso y el nivel de vibraciones y de ruido, en los sistemas de transmisiones.

- Evaluar el comportamiento del apareamiento entre dos engranajes obtenidos mediante diferentes procesos de elaboración y/o con diferentes tratamientos superficiales.

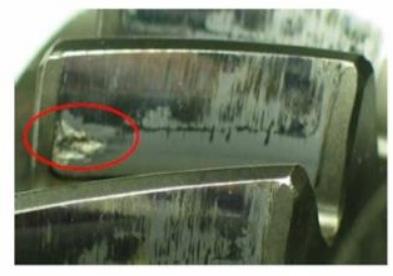

(a)

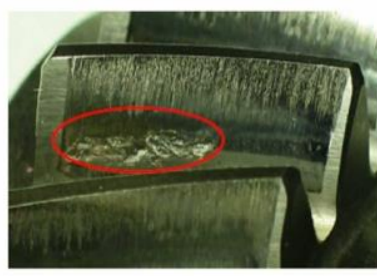

(b)
Figura 1 - Condiciones de picadura para engranajes con idénticas características y geometrías, excepto el método de elaboración (a) Rectificado (b) Sin rectificar [1].

Contar con bancos de ensayo para engranajes, permite además, la evaluación de determinados lubricantes, desarrollados por nuestra industria nacional, o de nuevos aditivos para estos, lo cual sería muy difícil y costoso de lograr en condiciones reales de explotación, pues pocas veces se dispone de las facilidades necesarias "in sito", para realizar, mediciones de torque, velocidad, vibraciones, espesor de la película y temperatura del lubricante. También resulta posible, validar experimentalmente, los resultados obtenidos de manera teórica, a través de cálculos matemáticos o mediante la aplicación de métodos de elementos finitos.

Se puede afirmar, que las instalaciones para la realización de ensayos, desempeñan un rol muy importante dentro del ciclo de desarrollo de cualquier producto, pero en el caso concreto de las transmisiones por engranajes, resultan indispensables para poder estudiar y predecir las posibles fallas que atentan contra el normal desempeño de estos sistemas, lo cual tributa a una mayor longevidad y fiabilidad durante la explotación de este tipo de transmisiones.

\section{FALLAS MÁS COMUNES EN LOS ENGRANAJES}

Una valoración completa y precisa de cualquier falla en engranajes, requiere un conocimiento previo de los modos básicos de fallas propios de estos elementos, sus causas y posibles soluciones. Con el objetivo de profundizar al respecto, se consultaron varios artículos [2-7] dedicados a este tema. Generalmente, las fallas principales de los dientes en engranajes, tienen un carácter de fatiga, como consecuencia de la acción periódica de la carga, dada la entrada sucesiva del diente en ciclos de trabajo y vacío. El deterioro de los dientes se manifiesta con aumento del nivel de ruido y sobrecalentamiento de la transmisión [8].

Existen múltiples factores que pueden incidir en el desencadenamiento de fallas en los sistemas de transmisión por engranajes, los cuales resultan necesario conocer, para poder controlar sus efectos negativos sobre estos fenómenos (Figura 2).

La norma ANSI/AGMA 1010-E95 [9] agrupa los principales modos de fallas en engranajes, en siete clases generales: Desgaste, Agarramiento, Deformación plástica, Fatiga por contacto, Agrietamiento, Rotura y Fatiga por flexión.

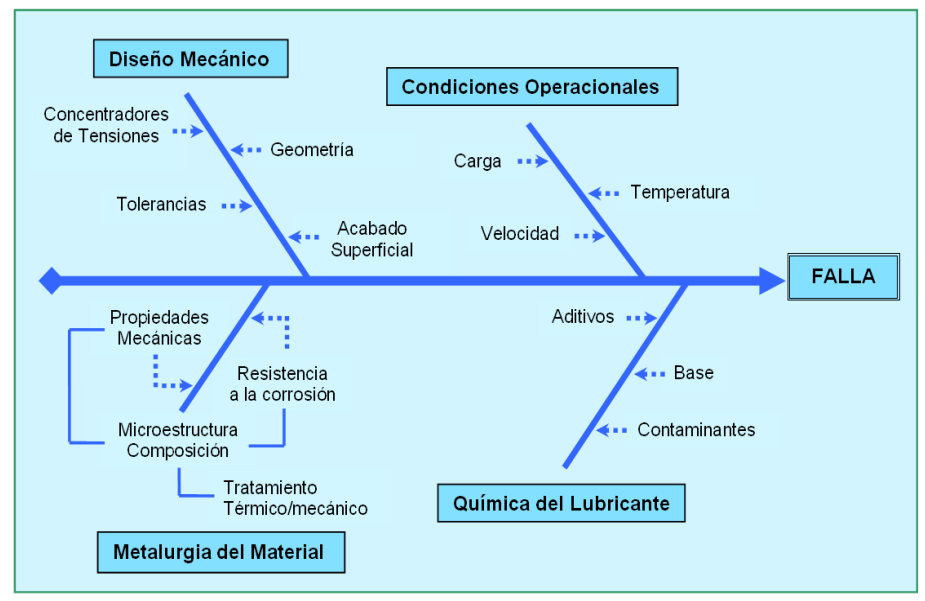

Figura 2-Factores que inciden sobre las fallas en engranajes [10]. 
Jorge L. Moya Rodríguez et al/ ITEGAM-JETIA Vol.02, Nº6, pp.111-126. Junho, 2016.

Las fallas casi nunca ocurren como un hecho aislado. Dos modos, o más, pueden ocurrir simultáneamente o en sucesión, y el modo de falla probable puede ser diferente al de la causa inicial [2].

Los dos modos de fallas más frecuentes en las transmisiones por engranajes y a cuya resistencia suelen ser verificados según los principales criterios de diseño, establecidos, incluso en las normas ISO 6336 [11][12], son la fatiga por contacto y la fatiga por flexión.

\section{1. FALLAS DE FATIGA POR CONTACTO.}

La falla de fatiga por contacto a picadura de las superficies útiles de los dientes es la causa principal que inutiliza las transmisiones por engranaje que trabajan con abundante lubricación.

La fatiga superficial puede ser advertida por la remoción de metal y la formación de cavidades. Estas pueden ser pequeñas $(0,38 \div 0,76 \mathrm{~mm})$ o grandes $(2 \div 5 \mathrm{~mm})$ y pueden crecer o quedarse del mismo tamaño [2].

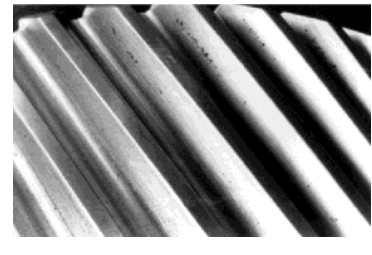

(a)

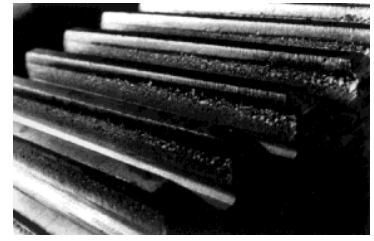

(b)
Figura 3 - Picadura: a) Inicial, b) Progresiva [9].

La picadura de la superficie de los dientes de acero empieza en los pies de los mismos, cerca de la línea polar. Se distingue la picadura inicial o limitada (Figura 3a) y la progresiva (Figura 3b). Si la dureza de las superficies de los dientes es $\mathrm{HB}<350$, entonces después de la fase inicial de funcionamiento de los dientes esta escoriación puede cesar. Si la dureza de la superficie de los dientes es HB > 350 la picadura limitada pasa, con frecuencia, a progresiva [8].

En las transmisiones abiertas, donde la lubricación es limitada, la picadura se observa muy raramente, pues la capa superficial, en la cual se producen las grietas iníciales, se desgasta antes de que tenga lugar el proceso de rotura por fatiga [8].

Cuanto más dura y fina sea la superficie útil de los dientes tanto mayor carga podrán soportar, sin que se produzca picadura [8].

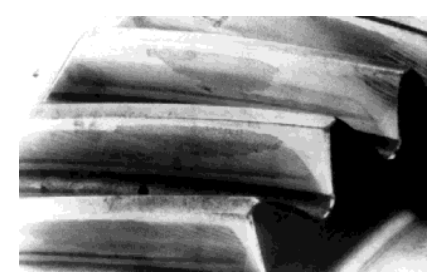

(a)

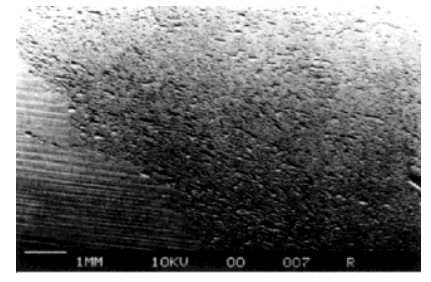

(b)
Figura 4 - Micropicadura, a.)Vista normal b.)Vista Ampliada [9].

La Micropicadura es un tipo de fatiga de contacto que aparece como un esmerilado a mancha gris bajo condiciones de una capa fina (Figura 4 a). Cuando es visto bajo aumento (Figura $4 \mathrm{~b}$ ), la superficie se muestra como un área de muy finos micro agujeros de alrededor de 2,5 $\mu \mathrm{m}$ de profundidad [2].

La resistencia a la picadura se puede elevar, mejorando las propiedades mecánicas de la superficie del diente, aumentando los radios de curvatura de los perfiles de los mismos en la zona de contacto y eligiendo correctamente el lubricante.

\section{II.2. FALLAS DE FATIGA POR FLEXIÓN}

La Falla de fatiga por flexión, consiste en una fractura total del diente (Figura 5), o de una parte considerable de este, motivada por la acción de tensiones cíclicas que exceden el límite de resistencia del material [2].

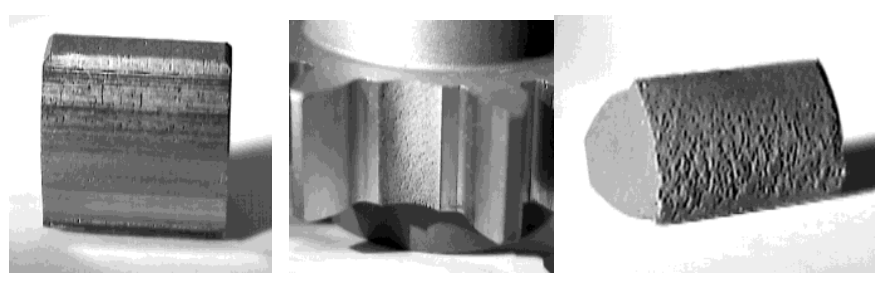

Figura 5 - Falla por rotura de un diente de engranaje [13].

La rotura por Flexión comienza con una grieta en la sección de la raíz y progresa hasta que el diente a parte de este, se rompe. Concentradores de tensión, tales como escalones o entalladuras en la raíz del diente, inclusiones no metálicas, pequeñas grietas provocadas por el tratamiento térmico, desgarraduras o huellas dejadas por las herramientas de corte, pueden condicionar la aparición de esta falla [2].

La resistencia de los dientes a la rotura se puede elevar, haciendo más firme la base del diente y disminuyendo la concentración de tensiones en torno a esta base, mediante el aumento de la curva de transición, un acabado minucioso de la superficie, la elevación de la rigidez de la transmisión, de la exactitud de fabricación y de las propiedades mecánicas del material de las ruedas. 
Jorge L. Moya Rodríguez et al/ ITEGAM-JETIA Vol.02, Nº6, pp.111-126. Junho, 2016.

\section{MÁQUINAS PARA EVALUAR EL CONTACTO EN TRANSMISIONES POR ENGRANAJES}

Atendiendo a los especímenes de ensayo utilizados, estas máquinas pueden ser clasificadas como tipo engranaje o tipo simulación, ya sean engranajes o elementos con una geometría básica simple, los que se someten a prueba, respectivamente [14].

\section{III.1. MÁQUINAS DE ENSAYO TIPO SIMULACIÓN}

Uno de los métodos más racionales para el estudio de la lubricación, el desgaste, y las formas de destrucción de los contactos metálicos lubricados altamente cargados, consiste en la simulación del contacto real empleando máquinas especialmente construidas para este fin [15].

Precisamente todas las instalaciones experimentales para estos estudios se basaron en la simulación del contacto de las transmisiones dentadas. Los primeros trabajos experimentales en la lubricación de los engranajes, estaban encaminados, fundamentalmente, hacia la determinación de su influencia en las transmisiones, sin entrar a analizar los aspectos relacionados con los fenómenos locales de las superficies lubricadas; algunos de estos trabajos se señalan en las referencias [16-18].

Al surgir la teoría de Martín [19] sobre la lubricación hidrodinámica en los engranajes y su desarrollo posterior sobre la base de los conceptos elasto-hidrodinámicos, se realizaron una serie de trabajos experimentales para dar respuestas a la contradicción existente entre la alta eficiencia observada en las transmisiones por engranajes y el pequeño espesor de la película lubricante que predecía dicha teoría.

En el año 1940, Beek, Givens y Smith [20] utilizaron la máquina de cuatro bolas (Figura 6) para la investigación de las propiedades de capa límite de lubricante. En 1949, Mckee, Swindells, White y Wayne [21] diseñaron una máquina de simulación, que denominaron SAE, formada por dos cilindros en contacto rotados a diferentes velocidades bajo carga, provocando condiciones de deslizamiento-rodadura típicas de los engranajes, para medir el desgaste de los rodillos.

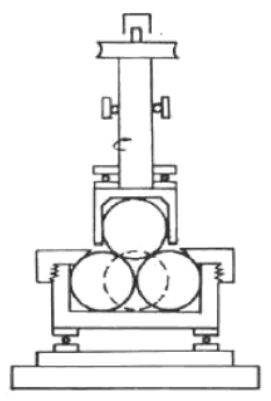

Figura 6 - Máquina de cuatro bolas [20].
Para el estudio de los contactos puntuales, Lane [22] en 1951 y Talian [23] en 1964, utilizaron la máquina de dos bolas cuyo principio aparece representado esquemáticamente en la Figura 7.

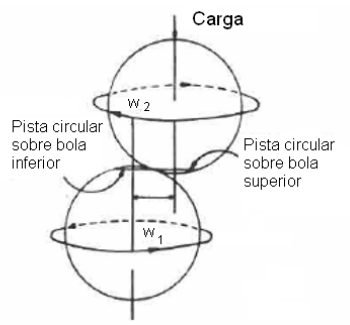

Figura 7 - Máquina de Lane y Tailan [22].

Crook [24], en el año 1957, construyó la máquina de cuatro discos que se muestra en la Figura 8.

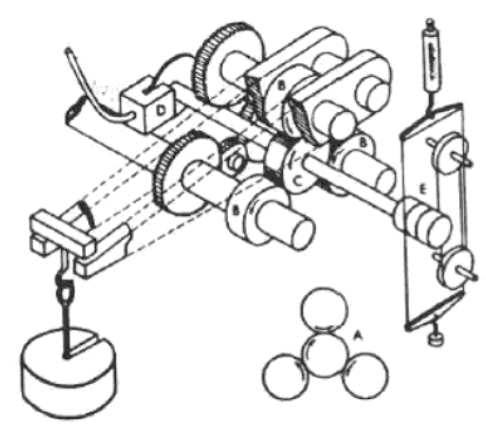

Figura 8 - Máquina de Crook [24].

Con el propósito de resolver las desventajas de la máquina de dos bolas, o sea, la elaboración de las esferas, Smith [25] en 1959 construyó la máquina de dos discos con ejes cruzados mostrada en la Figura 9.

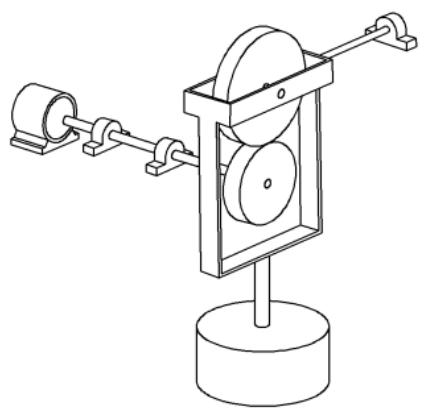

Figura 9 - Máquina de Smith [25].

En la Figura 10 se muestra la máquina de cilindros cruzados, la cual tiene el mismo propósito que la de dos discos, pero además permite evaluar el comportamiento del contacto, a todo lo largo de la superficie de ambos cilindros. 


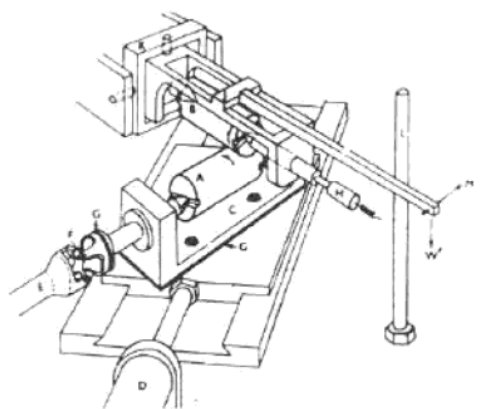

Figura 10 - Máquina de cilindros cruzados [25].

Sibbey y Orcutt [26] en 1961, diseñaron una máquina de dos discos rodantes a presión con sus ejes dispuestos paralelamente (Figura 11).

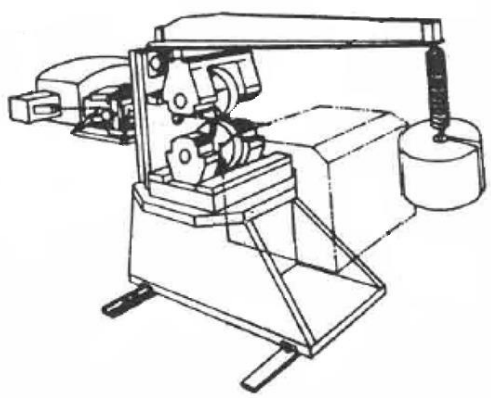

Figura 11 - Máquina de Sibbey y Orcutt [26].

Dowson y Longfield [27] en el año 1964, utilizaron un disco giratorio moviéndose dentro de una carcaza estacionaria de mayor diámetro. En la Figura 12 se muestra una variante de este tipo de máquina, diseñada por Archard y Kira [28], en la cual la carga es aplicada por medio del peso colocado en el extremo de una palanca rígida.

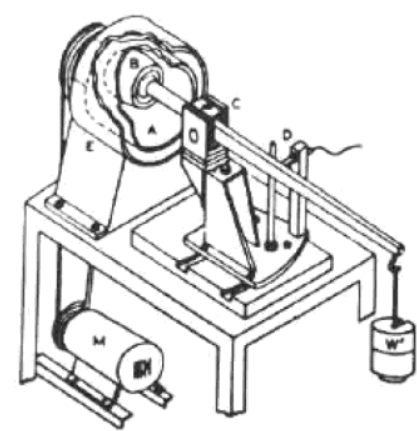

Figura 12 - Máquina de Archard y Kira [28].

En un trabajo conjunto realizado por profesores de la Universidad Central de las Villas y de las Universidades de Cienfuegos y Oviedo, se proyectó y construyó una máquina [15] para la simulación del contacto en las transmisiones por engranajes. En la Figura 13, se muestra un esquema de la máquina de rodillos con ejes paralelos que sirvió como base para su diseño, esta máquina está dirigida a la investigación de los diferentes modos de contacto, así como al estudio de los fenómenos relacionados con el deterioro de las superficies, (picadura, desgaste y agarramiento).

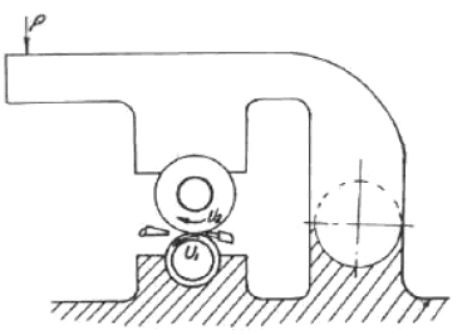

Figura 13 - Máquina de rodillos con principio de cascanueces [15].

En esta máquina se simula el contacto real entre dos evolventes, sustituyéndolo por el contacto entre dos rodillos en el punto deseado de la línea de engranajes. Para lograr simular exactamente las condiciones del engranaje a evaluar, se requiere que la carga específica en la máquina, y la velocidad de deslizamiento en ella, sean del mismo orden que en el engranaje real. La instalación contempla pasos por correas y por engranajes, de tal manera que se logren las relaciones deslizamiento-rodadura requeridas (Figura 14) [15].

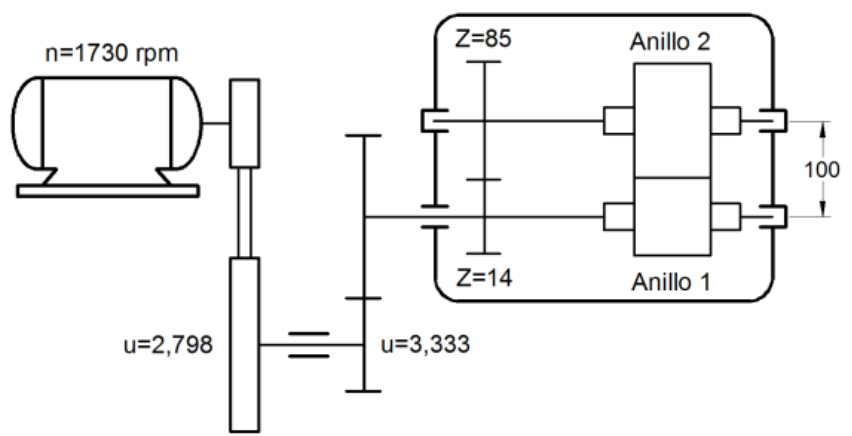

Figura 14 - Esquema cinemático de la máquina de simulación del contacto.

Las máquinas de ensayo del tipo simulación han demostrado tener una deficiente correlación con los resultados obtenidos durante la explotación real de los engranajes [14].

\section{III.2. MÁQUINAS DE ENSAYO TIPO ENGRANAJE}

Con las máquinas de ensayo del tipo engranaje, se consigue una reproducción más fiable de las condiciones de servicio reales. Generalmente son del tipo de potencia circulante bajo el principio conocido como Four Square. 
La opinión general de la industria apunta hacia la tendencia del empleo de máquinas de ensayo tipo engranajes, pues la correlación con el comportamiento real en servicio, ha sido mejor utilizando engranajes como especímenes de ensayo [14].

Los especímenes de ensayo pueden ser engranajes reales cuya forma, dimensiones y material pueden estar normalizados para la evaluación de lubricantes, pero pueden ser alterados para evaluar específicamente determinados requerimientos de servicio que se deseen.

Un modo aparentemente simple de diseñar tales máquinas consiste en colocar los engranajes de ensayo entre un motor y un freno como se muestra en la Figura 15.

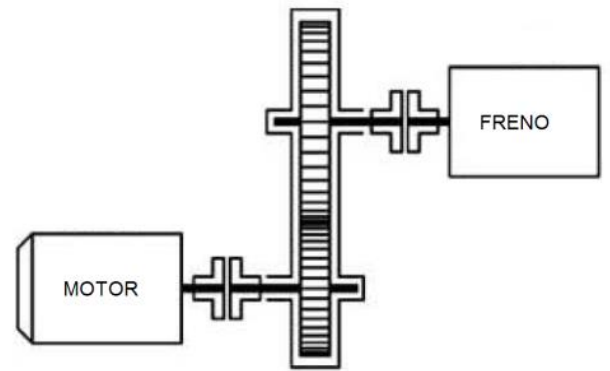

Figura 15 - Máquina para el ensayo de engranajes con lazo de potencia abierto [14].

Sin embargo la implementación de tales diseños resulta limitada debido a las siguientes desventajas:

* El motor debe suministrar toda la potencia necesaria para garantizar la carga de ensayo a la cual se desean probar los engranajes.

* Alto costo de instalación y alto consumo de energía.

Un modo más eficiente para aplicar carga sobre engranajes de ensayo consiste en la propia inclusión de estos dentro de un lazo o circuito cerrado de transmisión de potencia, el cual puede ser eléctrico o mecánico [14], como se muestra en la Figura 16.

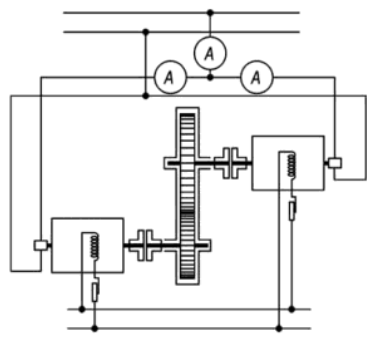

(a)

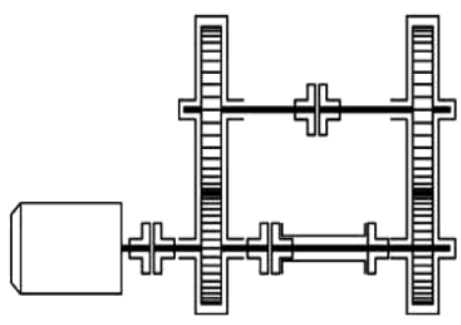

(b)
Figura 16 - Máquina para el ensayo de engranajes con lazo de potencia cerrado, a) Eléctrico, b) Mecánico [14].

En el primer caso un motor eléctrico acciona el eje de entrada de la caja de engranajes sometidos a ensayo, el eje de salida es acoplado a un generador que retroalimenta energéticamente al sistema, sin embargo el gran tamaño, tanto del motor como del generador, constituyen su principal limitación, ya que estos deben ser seleccionados en concordancia con la potencia de ensayo máxima requerida, la cual es generalmente más alta que la nominal. En el segundo caso, los ejes de entrada y salida de dos cajas de engranaje que tienen exactamente la misma relación de transmisión son conectadas unas a otras mediante ejes intermedios, conformando un lazo cerrado. Un dispositivo para la aplicación de torque puede ser incluido en dicho lazo y el sistema se hace girar utilizando un motor externo. En condiciones estables este solo debe suministrar las pérdidas de energía total del sistema y por consiguiente este rango de potencia es mucho más bajo que la potencia que realmente carga los engranajes.

Las ventajas de este tipo de máquinas de ensayo son:

* Significativa reducción del costo de instalación, ya que no se requieren ni freno ni generador y el motor de accionamiento está seleccionado en concordancia con las pérdidas de potencia total del sistema.

* El consumo de energía es mucho menor que en los sistemas anteriores.

* Permite la determinación de la eficiencia del sistema de engranajes.

La configuración de máquina anteriormente descrita se conoce como de potencia circulante, y su principio de funcionamiento se denomina en inglés como Four-Square.

\section{III.2.1 SISTEMAS DE POTENCIA CIRCULANTE O FOUR SQUARE}

Los sistemas de potencia circulante o Four-Square son una configuración mecánica, utilizada básicamente en el ensayo a fatiga de componentes [29].

Con el objetivo de explicar en qué consiste este principio se le denominará cadena de torsión, a la unión de dos elementos: un transmisor de torque (engranaje ó polea) y un generador de torque (motor ó actuador hidráulico). Esta unión se materializa mediante un elemento intermedio, que se encarga de transmitir el momento torsor desde el elemento generador hasta el transmisor. En la Figura 17 a se muestra una cadena esquematizada y pueden identificarse los elementos constituyentes.

Las dos cadenas se unen por sus respectivos transmisores de torque (Figura 17 b). El esquema del Four-Square se completa añadiendo dos transmisores de torque (con la misma relación de transmisión que los ya existentes), (Figura 18).

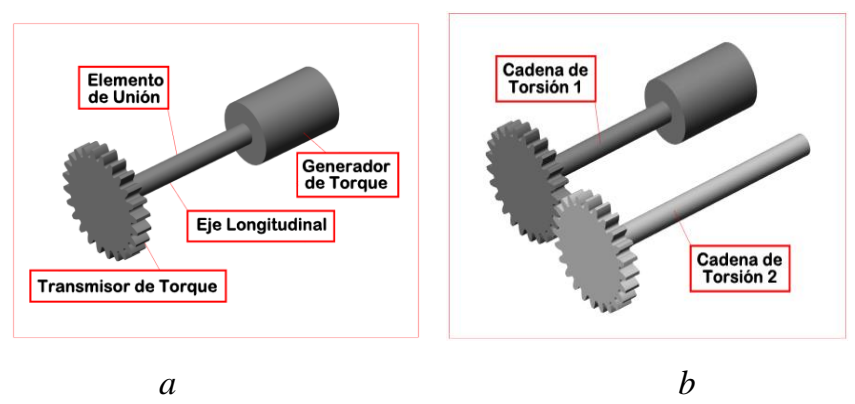

Figura 17 - a) Elementos componentes de la cadena de torsión, b) Cadenas de torsión unidas. 
Los elementos ensayados tienen en común que durante su servicio, trabajan sometidos a momentos torsores, mientras giran a una determinada velocidad angular. Los especímenes ensayados pueden ser los definidos como transmisores de torque, o formar parte de lo que se ha llamado elemento de unión. Un ejemplo del primer tipo es el ensayo de engranajes a fatiga. En este caso, las dos cadenas se someten al momento recirculante entre elementos.

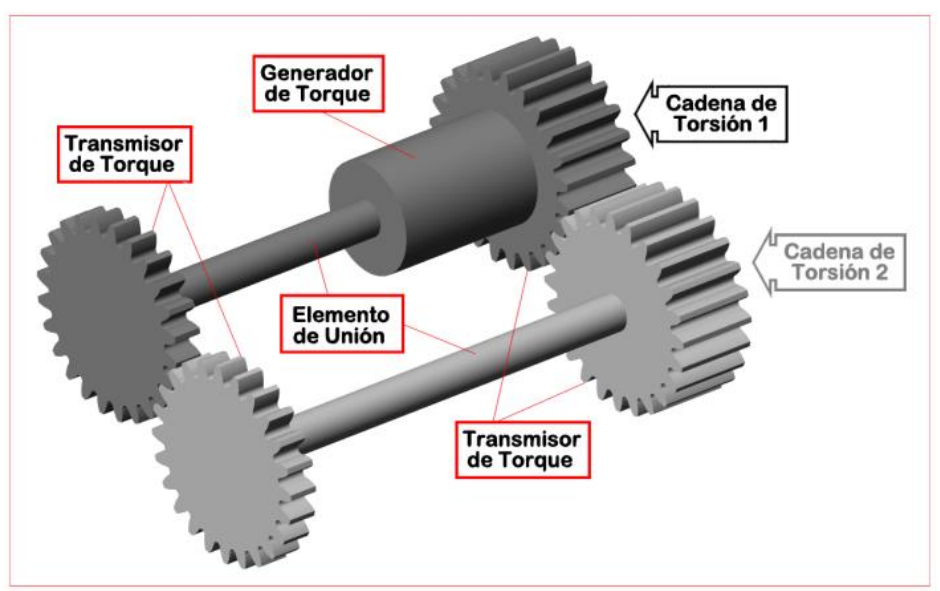

Figura 18 - Esquema general del mecanismo de potencia circulante.

\section{III.2.2 DISPOSITIVOS UTILIZADOS PARA GENERAR TORQUE EN LAS MÁQUINAS DE ENSAYO}

De acuerdo con el modo mediante el cual se aplica el torque de carga, las máquinas de ensayo para engranajes de potencia circulante pueden ser clasificadas en sistemas mecánicos e hidráulicos [14].

\section{III.2.2.1 SISTEMAS MECÁNICOS PARA LA APLICACIÓN DE TORQUE}

El acoplamiento de embrague para la aplicación del torque en máquinas como las del tipo FZG, resulta simple y fiable, sin embargo, tiene la desventaja que el torque aplicado no puede ser controlado durante el ensayo. Para variar el torque de ensayo durante su realización sin tener que interrumpirlo, para poder simular con precisión las condiciones de carga reales, es necesaria la utilización de un dispositivo adicional. Tales dispositivos emplean frecuentemente trenes de engranajes planetarios, Lanahan [30], Klinger [31], Langenbeck [32] and Basedow [33] presenteron sistemas de este tipo.

El más simple de estos, consiste en un tren de engranajes planetarios simple, el cual es mostrado esquemáticamente en la Figura 19.

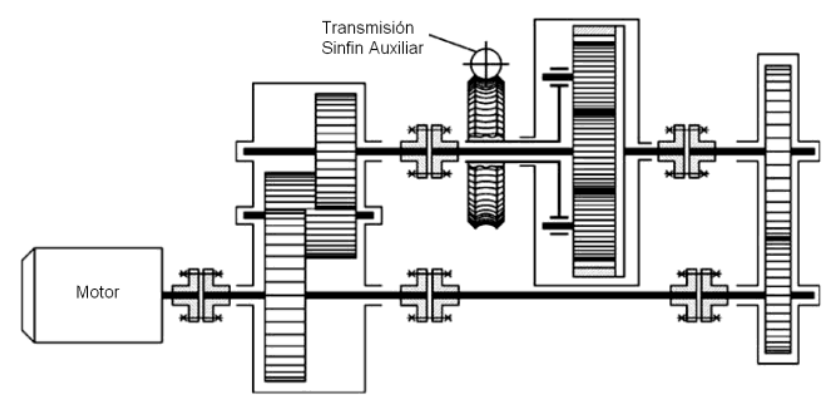

Figura 19 - Sistema planetario simple utilizado para imponer el torque de ensayo [30].

El torque es aplicado al cargador planetario mediante una transmisión sinfín auxiliar durante el ensayo, operada manualmente o controlada numéricamente mediante un servo motor. El torque de ensayo resulta de la diferencia en la dirección de rotación y la relación entre el engranaje sol y el anillo dentado interior.

En la Figura 20 se muestra un desarrollo adicional que emplea un tren de engranajes planetarios doble sin anillos dentados interiores. Los números de dientes de los engranajes son seleccionados de tal modo que para un ángulo de rotación dado del cargador planetario, los engranajes sol rotan en la misma dirección pero a diferentes ángulos, aplicando de este modo el torque de ensayo. Su principal desventaja es que no pueden ser usados, para determinar perdidas de potencia en cajas de engranajes.

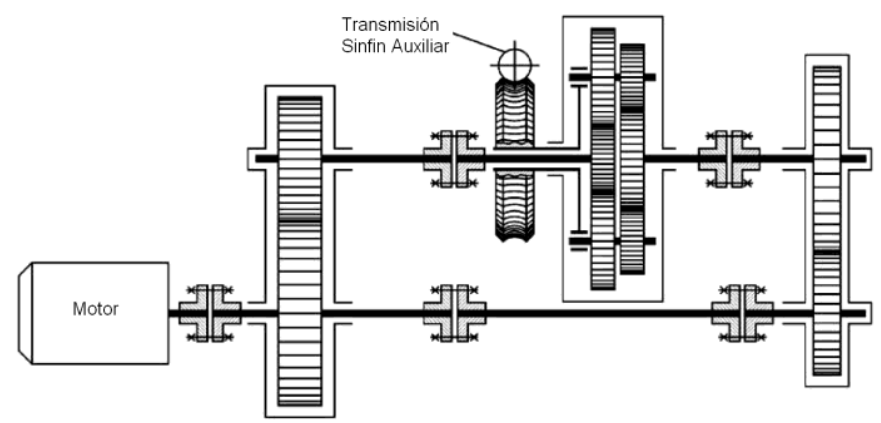

Figura 20 - Sistema planetario doble sin anillo dentado interior, utilizado para establecer el torque en el sistema de ensayo [31].

Para superar esta desventaja Gruscka y Herrmann [34] propusieron incluir en el lazo de potencia otro sistema planetario doble idéntico (Figura 21).

En esta configuración, ambas cajas de engranajes de ensayo pueden ser idénticas, sin embargo, la potencia fluye a través de los trenes de engranajes planetarios y causa pérdidas adicionales haciendo por lo tanto más difícil de obtener las pérdidas de potencia de una sola caja de engranaje de ensayo. 


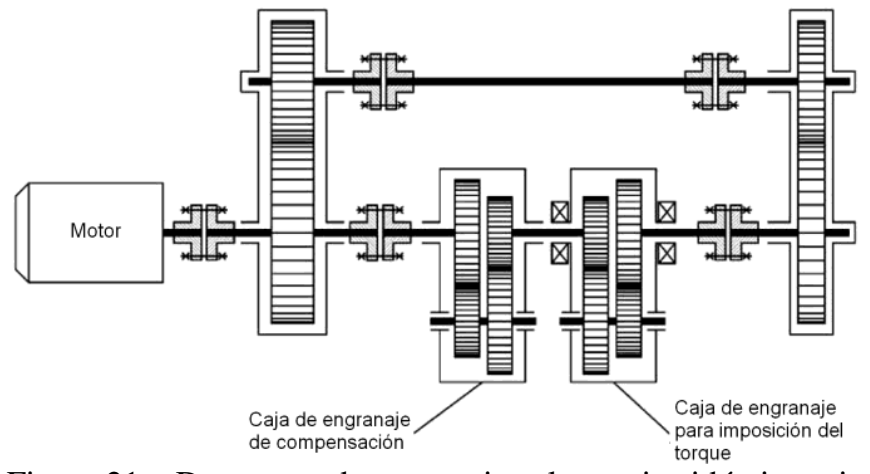

Figura 21 - Dos trenes de engranajes planetarios idénticos sin anillos dentados interiores para imponer el torque y compensar la velocidad rotacional respectivamente [34].

En lugar de utilizar trenes de engranajes planetarios, Musser [35] y Schröder [36] presentaron otro dispositivo que utiliza una transmisión harmónica (Figura 22).

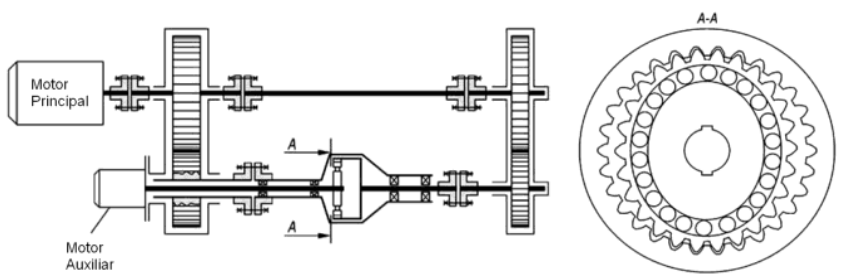

Figura 22 - Transmisión harmónica utilizada para imponer torque de ensayo [35].

El torque de ensayo se obtiene a partir de la diferencia en los ángulos de rotación del anillo dentado exterior del rodamiento y el engranaje interior. Una desventaja de este sistema estriba en que las pérdidas de potencia del motor auxiliar son generalmente desconocidas y perjudica el nivel de precisión. en la determinación de la eficiencia de una caja de engranaje sometida a ensayo.

Una variante de este diseño, basada en el mismo principio, fue presentada por Brüggemann [37], la cual emplea una transmisión cicloidal (Figura 23), de esta manera se extiende significativamente los valores máximos del torque de ensayo.

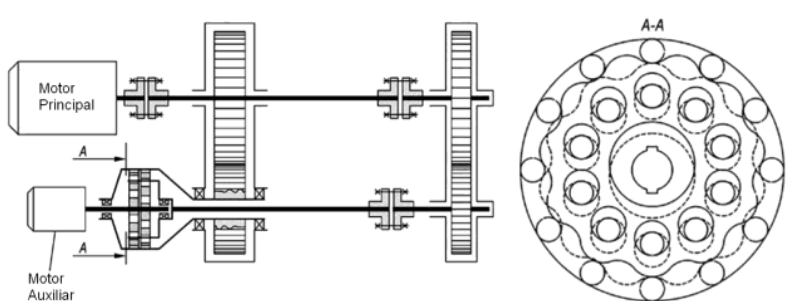

Figura 23 - Transmisión cicloidal utilizada para imponer torque de ensayo [37].

Otro interesante modo de aplicar carga, consiste en incluir dentro del lazo de potencia, un par de engranajes adicional que genere el torque de ensayo cuando este es movido en dirección transversal, como se muestra en la Figura 24, Harald [38] y Yano
[39] presentaron aparatos basados en este concepto, los que poseen como ventaja principal, que permiten rápidas variaciones del torque de ensayo.

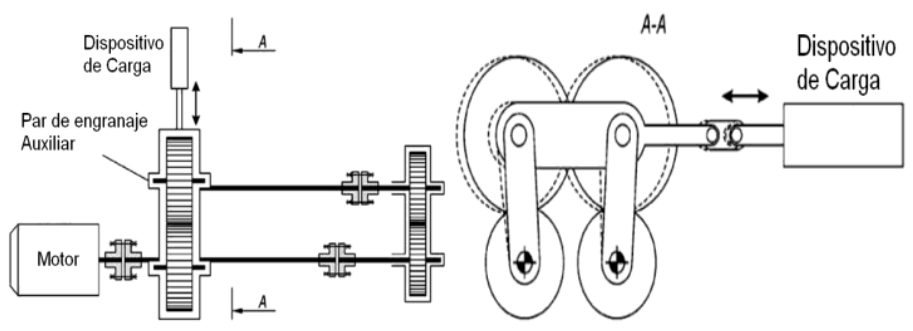

Figura 24 - Aplicación del torque de ensayo mediante colocación de un par de engranajes auxiliares en dirección transversal [38].

Otra alternativa fue intoducida por Bader [40] y consiste en una máquina conformada por dos cajas de engranajes conectadas por dos árboles con junta universal (Figura 25), una de las cajas de engranajes se rota sobre un eje paralelo al árbol de la transmisión, aplicando de esta manera el torque de ensayo.

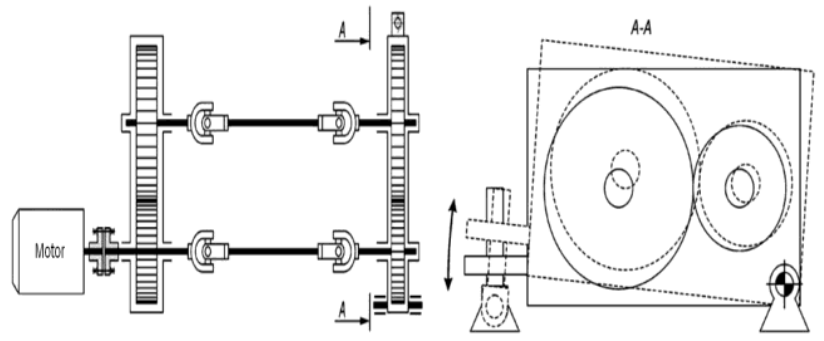

Figura 25 - Aplicación del torque mediante rotación de la caja de engranajes completa [40].

\section{III.2.2.2 SISTEMAS HIDRÁULICOS PARA LA APLICACIÓN DEL TORQUE}

Varios diseñadores reemplazaron el simple acoplamiento de carga de los sistemas mecánicos, por un torque hidráulico aplicando dispositivos para ajustar el torque de ensayo mediante el control de la presión hidráulica.

Uno de los primeros intentos de diseño de tales dispositivos fue presentado por Collins [41] (Figura 26), cuenta con un árbol intermedio incluido dentro del lazo de potencia, el cual está provisto en ambos extremos de un estriado helicoidal que tiene direcciones opuestas y se encuentra montado sobre rodamientos dentro de una caja que fue diseñada para operar como un pistón hidráulico bi-direccional.

Aplicando presión en uno de los compartimentos, se aplica una carga axial sobre el árbol intermedio y el torque de ensayo se genera gracias a los estriados helicoidales, como la fricción en los flancos de los dientes del árbol ranurado helicoidal es proporcional al torque aplicado, se introduce una dificultad para controlar el torque solo mediante la presión, se hace necesario montar un medidor de torque, para determinar su magnitud real, con precisión. 


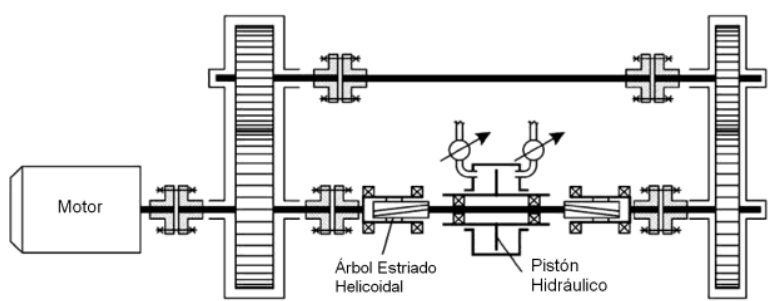

Figura 26 - Árbol estriado helicoidal montado en el interior de un pistón hidráulico [41].

Basado en el mismo principio de operación, Hennings [42] diseñó un acoplamiento generador de torque, consistente en un tambor y un disco provisto de un engranaje helicoidal, el cual es empujado por un pistón hidráulico, como se muestra en la Figura 27. Sin embargo, este diseño tiene las mismas desventajas que el anterior.

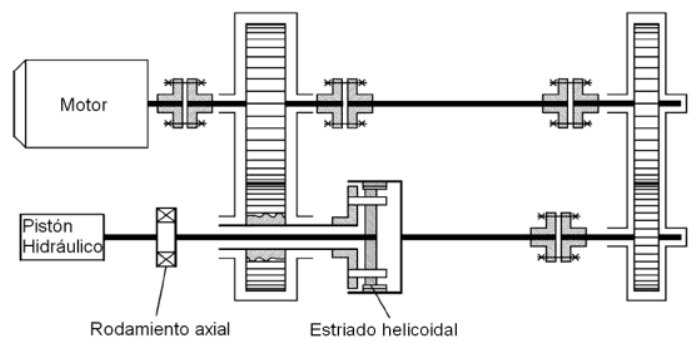

Figura 27 - Aplicador de torque con estriado helicoidal, controlado hidráulicamente [42].

En otra variante para aplicar torque de ensayo mediante la utilización de cilindros hidráulicos, Schneider [43] propuso montar un cilindro con un extremo conectado a una brida ensamblada sobre el árbol del engranaje. Mientras el otro extremo se conecta mediante un pasador colocado en el propio engranaje, como se muestra en la Figura 28. En este diseño el torque de ensayo ya no es afectado por la fricción.

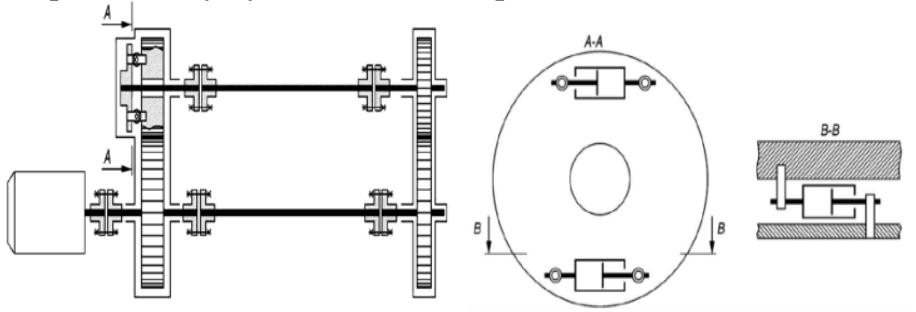

Figura 28 - Cilindros hidráulicos ubicados circunferencialmente para aplicar el torque [43].

En otro dispositivo para la aplicación de torque Shipley [44] introdujo un tambor y un rotor provisto con aletas radiales formando cámaras de presión (Figura 29), el torque de ensayo es generado mediante la presión de aceite en las cámaras. Un diseño apropiado minimiza la fricción y el torque de ensayo puede ser controlado de manera precisa mediante la presión. Este concepto ha sido probado de manera satisfactoria. Se encuentra comercialmente disponible para torques de hasta $8000 \mathrm{Nm}$.
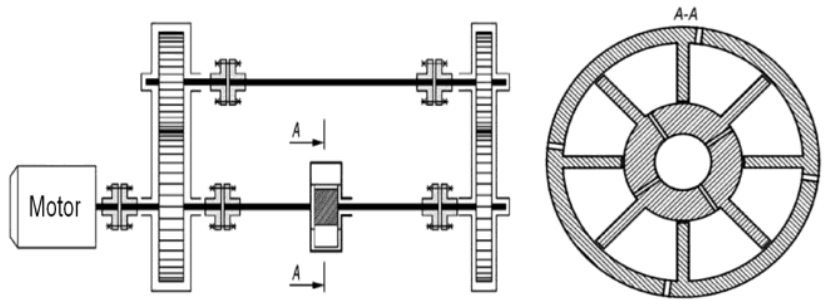

Figura 29 - Cilindro hidráulico rotatorio utilizado para aplicar torque de ensayo [44].

Kugler [45], en una variante más compacta, diseñó un cilindro rotatorio hidráulico, construido en el interior de uno de los engranajes fijos (Figura 30).

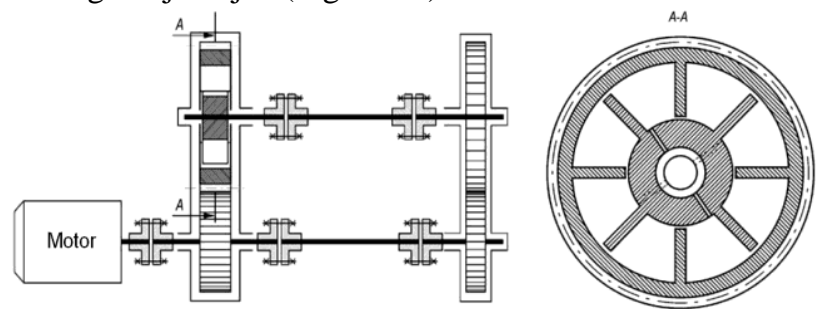

Figura 30 - Cilindro hidráulico rotatorio diseñado dentro del propio cubo del engranaje [45].

Un novedoso sistema para aplicar torque ha sido propuesto recientemente por Mihailidis [14, 46], cuyo componente principal es un tren de engranajes planetarios diseñado originalmente por Wolfrom [47], la Figura 31 muestra el concepto sobre el que se sustenta este sistema, el cual posee una elevada relación de transmisión, que permite aplicar elevados torques de ensayo, empleando motores de pequeño tamaño. El motor auxiliar opera solamente mientras se aplica o varía el torque de ensayo. El tren de engranaje planetario completo, incluyendo el motor fuera de operación, rota como un bloque. Por consiguiente, este sistema puede ser utilizado para obtener la eficiencia en cajas de engranajes mediante la simple medición del torque aplicado por el motor principal, toda vez que no ocurren pérdidas de potencia en el sistema de aplicación de torque, el cual opera como un árbol rígido.

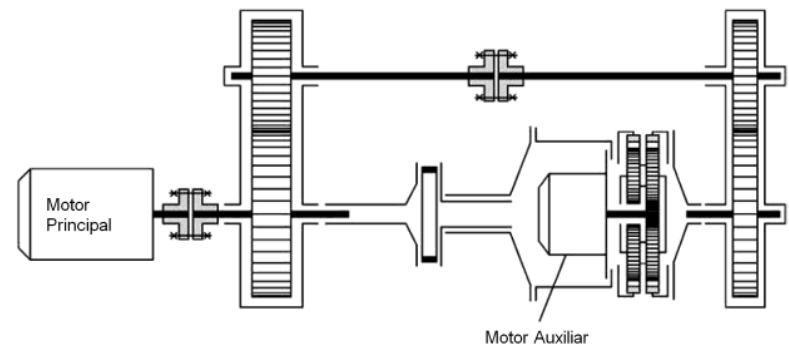

Figura 31 - Sistema de aplicación de torque propuesto recientemente por Mihailidis [14].

Una limitación de este sistema es que no permite realizar cambios de carga rápidos, que resultan necesarios para simular operaciones bajo fuertes choques. 
Jorge L. Moya Rodríguez et al/ ITEGAM-JETIA Vol.02, Nº6, pp.111-126. Junho, 2016.

\section{III.2.3 MÁQUINAS PARA EL ENSAYO DE ENGRANAJES A LA FATIGA SUPERFICIAL}

En la norma AGMA 925-A03 [48] de la Asociación Americana de Fabricantes de Engranajes, conocida por sus siglas en inglés AGMA, se hace referencia a las principales máquinas utilizadas para el ensayo de engranajes: NASA, FZG, Ryder e IAE, coincidiendo con los resultados obtenidos en las búsquedas realizadas sobre el tema, pues son precisamente estas las más se citadas, en las publicaciones referentes a trabajos investigativos realizados con engranajes. Sus principales características y aspectos de mayor interés, se exponen a continuación.

\section{III.2.3.1 MÁQUINA PARA EL ENSAYO DE ENGRANAJES NASA}

Esta máquina (Figura 32) ha sido desarrollada por el Centro de Investigación sobre Engranajes de la Administración Nacional para la Aeronáutica y el Espacio de los Estados Unidos (NASA), por sus siglas en inglés.
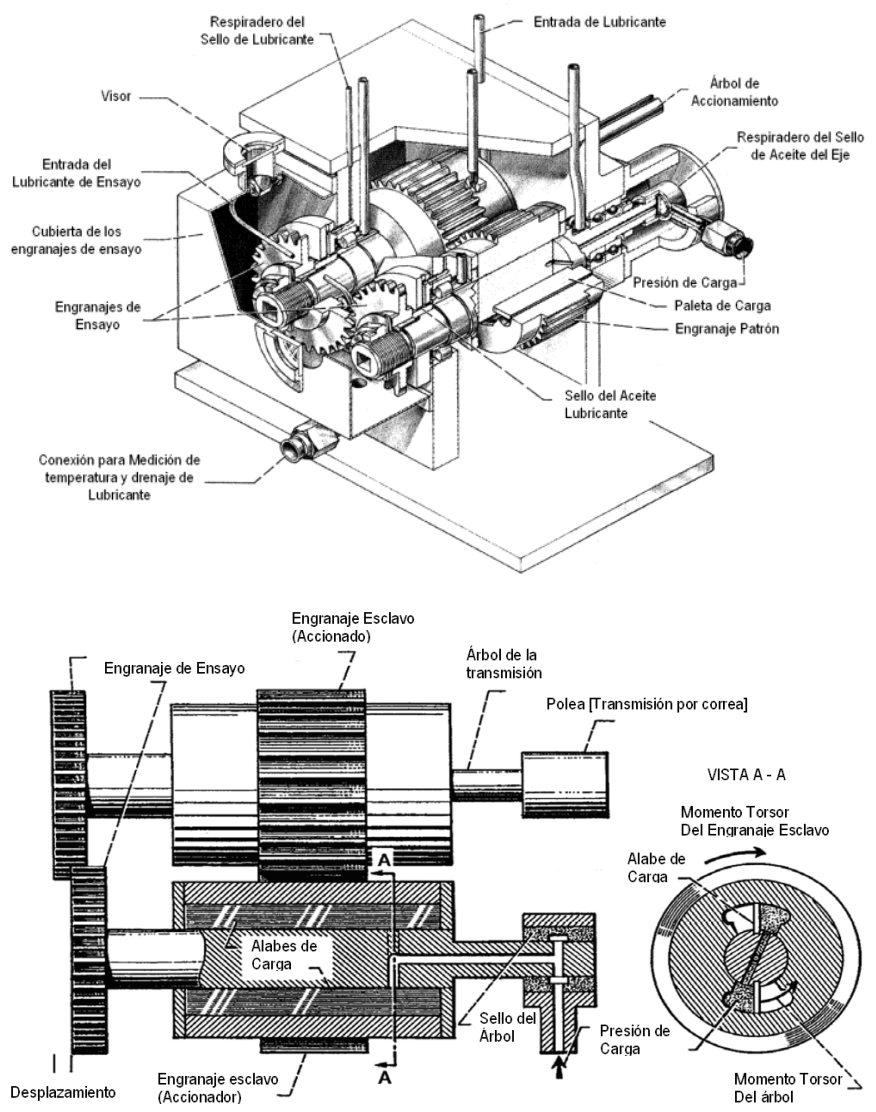

Figura 32 - Máquina para ensayo de engranajes NASA [49].

$\mathrm{Su}$ funcionamiento se basa en el principio de potencia circulante, puede ser puesta a girar sin carga mediante un sistema motriz, dotado de un motor eléctrico y una transmisión por correa, que le permite alcanzar una frecuencia de rotación de hasta $10000 \mathrm{rpm}$, para una velocidad circunferencial de $46,5 \mathrm{~m} / \mathrm{s}$, el sistema hidráulico puede alcanzar una presión máxima de $690 \times 10^{4} \mathrm{~N} / \mathrm{m}^{2}$, permitiendo establecer una capacidad de carga máxima de $6672 \mathrm{~N}$ y obtener tensiones de contacto cercanas a los $2 \mathrm{GPa}$ sobre la superficie del diente del engranaje sometido a ensayo [49].

Un sistema hidráulico es el encargado de proveer la presión de trabajo sobre la paleta de carga, localizada en el interiorx de uno de los engranajes patrones, lo cual genera un momento torsor que se recircula a través de los elementos de la transmisión y permite alcanzar el nivel de tensión requerida para el ensayo, sobre los dientes del engranaje, mediante ajuste de la presión hidráulica.

Los engranajes son probados con un desplazamiento de las caras que permite alcanzar la tensión de contacto deseada, a costa de un menor torque, posibilitando ademas realizar 4 pruebas por cada par de engranajes [49].

\section{III.2.3.2 MÁQUINA PARA EL ENSAYO DE ENGRANAJES FZG}

Esta es una de las máquinas de ensayo de engranajes (Figura 33) más utilizadas, con más de 500 unidades en explotación alrededor del mundo en el año 2008, fue desarrollada por la Universidad Técnica de Munich, Alemania, para su centro de investigación sobre engranajes, conocido por sus siglas en alemán FZG (Forschungsstelle für Zahn-räder und Getriebebau) [50], que dan nombre a la máquina.

Cuenta con dos cajas de engranajes, en una se encuentra montado el par de engranajes fijos de la máquina, y en la otra los de ensayo, ambos pares con la misma relación de transmisión. El árbol más lento posee un elemento intermedio para la medición del torque. El eje rápido está conectado mediante un acoplamiento embridado, que permite a ambos extremos rotar uno con respecto al otro, a través de este acoplamiento se aplica el torque de ensayo mediante una palanca y un contrapeso, cuya activación se ejecuta a través de un pasador de bloqueo que impide el movimiento relativo entre las bridas, transmitiendo asî el torque aplicado [51].

Esta máquina de ensayo es muy utilizada para determinar la capacidad de carga por desgaste de aceites lubricantes y grasas, y la influencia de estos, sobre el coeficiente de fricción, la eficiencia, y la formación de micro-picadura y picadura.

Establece una presión de contacto máxima entre los dientes de $2 \mathrm{GPa}$, alcanza unos $530 \mathrm{Nm}$ de torque y una velocidad de rotación de $2250 \mathrm{rpm}$ [48]. Un control de nivel de vibración permite la detección de astillado u otros daños severos.

Los laboratorios de tribología frecuentemente utilizan estas máquinas para ensayar materiales también, utilizando para ello el mismo tipo de lubricante, lo cual ofrece resultados que dependen únicamente de las características del propio engranaje, cuando son producidos con diferente material o modo de elaboración [13]. También resultan ampliamente utilizadas para determinar la capacidad de carga al agarramiento [52-56] y el 
comportamiento respecto al desgaste [57] de aceites y grasas, así como la influencia de estos sobre el coeficiente de fricción y la eficiencia $[58,59]$ y sobre la formación de micro-picadura [60] y picadura [61, 62], el mismo concepto de diseño ha sido utilizado en máquinas para el ensayo de engranajes hipoides [63] y helicoidales cruzados [64].
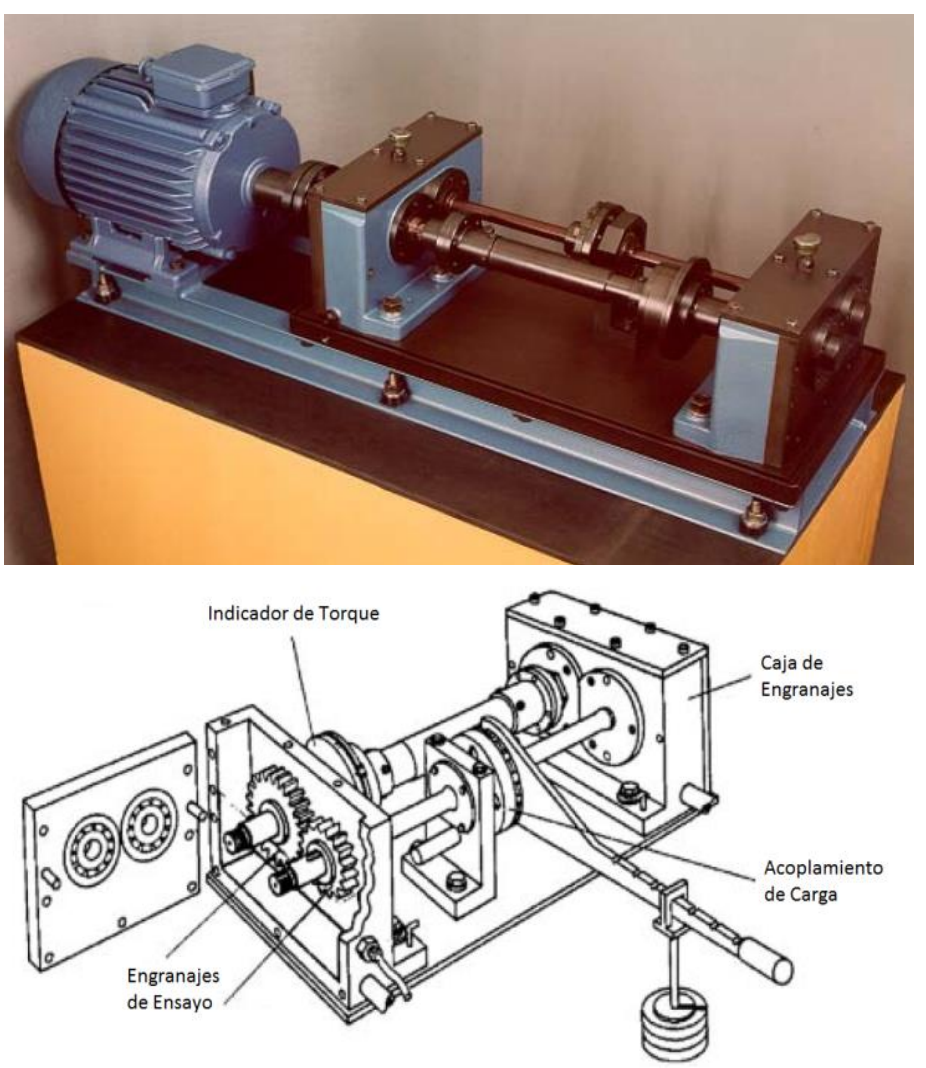

Figura 33 - Vista general y esquema de la máquina de ensayo FZG [65].

La máquina FZG tiene limitaciones tales como el bajo límite superior de tensiones de contacto en el diente engranado (2000 MPa), y los intervalos excesivamente amplios en el cambio de las cargas aplicadas, lo cual disminuye el nivel de precisión.

\section{III.2.3.3 MÁQUINA PARA EL ENSAYO DE ENGRANAJES IAE}

Esta máquina de fabricación inglesa, es del tipo de potencia circulante, los engranajes de ensayo son del tipo cilíndrico de dientes rectos [66]. Las cargas son aplicadas por medio de un brazo-palanca, su velocidad oscila entre 4000 y $6000 \mathrm{rpm}$ y el torque entre 20 y $407 \mathrm{Nm}$ [48], lo cual genera una carga máxima sobre los engranajes de ensayo que permite alcanzar una alta presión especifica en la zona de contacto de hasta $35600 \mathrm{kgf} / \mathrm{cm}^{2}$, permitiendo evaluar lubricantes para transmisiones universales e hipoides con aditivos de extrema presión muy activos [67].
Algunos autores $[68,69]$ refieren las notables ventajas que posee esta máquina para evaluar las propiedades antiagarramiento de lubricantes para transmisiones.

\section{III.2.3.4 Máquina para el ensayo de engranajes Ryder}

Se utiliza para evaluar la capacidad de carga y propiedades anti-agarramiento de lubricantes para la aviación, de ahí su elevada velocidad de operación de hasta $10000 \mathrm{rpm}$ y un rango de torque de 0 a $270 \mathrm{Nm}$ [48].

Está compuesta por una sola caja de engranajes con dos árboles conectados por dos pares de engranajes cilíndricos, los cuales tienen exactamente la misma relación de transmisión y forman un lazo de potencia cerrado (Figura 34). En la configuración original los engranajes estrechos eran rectos mientras los anchos eran helicoidales. La característica principal de esta instalación es la aplicación de carga a los engranajes de ensayo, mediante movimiento axial de un engranaje helicoidal relativo al otro, debido a la acción de una presión de aceite conocida, sobre el pistón ubicado en el interior del cubo de uno de los engranajes helicoidales [66]. Partiendo del ángulo de la hélice y el área de la cámara presurizada, es posible calcular la carga tangencial. La máquina de ensayo Ryder ha sido ampliamente adoptada y aun está siendo utilizada en ensayos de lubricantes y engranajes especialmente en los Estados Unidos [70]. Esta permite variar de manera efectiva el torque de ensayo durante la operación mediante el control de la presión hidráulica, pero no es posible determinar la eficiencia de un solo par de engranajes ni pueden ser probadas cajas de engranajes completas [14].

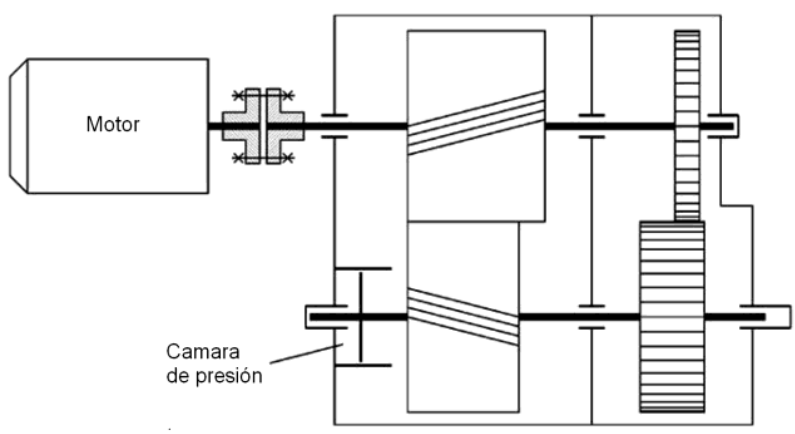

Figura 34 - Máquina para ensayo de engranajes Ryder [71].

\section{III.2.4 MÁQUINAS PARA EL ENSAYO DE ENGRANAJES A LA FATIGA POR FLEXIÓN}

La rotura del diente es la forma más catastrófica de falla en engranajes, por lo que una parte considerable de los programas de ensayo de engranajes están dedicados a obtener suficiente información para minimizar su ocurrencia en servicio [72]. 
Existen dos grupos principales de máquinas destinadas a la realización de ensayos en engranajes a la fatiga por flexión, las ya conocidas de potencia circulante o tipo Back-to-Back y las de tipo Pulsador, las primeras son rotatorias y ya han sido analizadas con anterioridad, aunque más adelante se detallan algunas peculiaridades que caracterizan su uso en los ensayos de engranaje a la fatiga por flexión y las segundas son estáticas y se utilizan para realizar ensayos sobre diente único.

\section{III.2.4.1 MÁQUINAS TIPO PULSADOR}

Este tipo de ensayo es utilizado para general datos de fatiga por flexión en cantidades estadísticamente significativas, a un costo económico comparativamente bajo. Los dientes son ensayados cada uno de manera independiente, aplicando la carga de ensayo sobre un punto fijo del único diente sometido a prueba [72].

El ensayo es generalmente realizado en una máquina electro-hidráulica universal, servo-controlada, donde el engranaje es rígidamente soportado sobre un eje, colocado en un aditamento especialmente diseñado, de manera que un diente pueda ser sometido a la carga de ensayo, mientras otro soporta la reacción [73].

En la Figura 35 se muestra una configuración típica de este tipo de instalación, diseñada para engranajes cilíndricos de dientes rectos, con varios dientes removidos para facilitar el acceso a los dientes de ensayo y de reacción. La carga se aplica a través de un vástago que entra en contacto con el punto más alto del diente sometido a ensayo. La reacción es soportada a través de otro vástago en contacto con el diente de reacción, en su punto más bajo. La capacidad de carga típica para ensayos a la fatiga en estos equipos oscila entre 45 y $90 \mathrm{kN}$ [72].
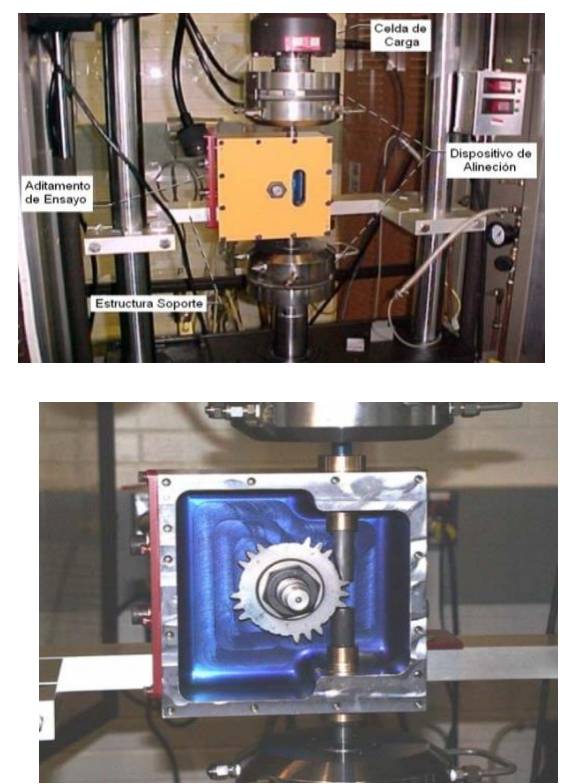

Figura 35 -Máquina de ensayo a la fatiga por flexión sobre diente único [74].
A pesar de que en el ensayo sobre diente único, no se reproducen las condiciones reales de engranaje, existen varias razones [75] que hacen apropiado a este tipo de ensayo, para evaluar el comportamiento de los engranajes a la fatiga por flexión:

$>$ Elimina la implicación que sobre el ensayo pudieran tener ciertas variables de la máquina, tales como desgaste de los árboles, rodamientos y otras partes de las maquinas rotatorias, que frecuentemente tienden a confundir los resultados de la prueba, en este ensayo el diente sometido a prueba recibe la carga directamente.

$>$ Elimina las posibles influencias que las variables del engranaje pudieran provocar. El efecto de las desviaciones en la línea de paso, el huelgo entre dientes y los errores del perfil de involuta, son eliminados debido a que el contacto ocurre siempre en la misma posición.

$>$ Pueden ser utilizadas varios tipos de máquinas estándar de ensayo a la fatiga.

$>$ El ensayo puede ser ejecutado continuamente hasta que la máquina sea automáticamente detenida cuando ocurra la falla del diente.

$>$ Permite la medición de las tensiones por flexión en la raíz del diente en pleno proceso de operación, si los dientes del engranaje son suficientemente grandes.

$>$ Elimina fallas indeseadas al no existir movimiento relativo contra el diente sometido a ensayo, por lo que solo pueden ocurrir fallas por fatiga a la flexión, esto posibilita la generación de datos por concepto de este tipo de falla, a ciclos comparativamente altos, sin riesgo de perder los ensayos debido a otras fallas.

\section{III.2.4.2 MÁQUINAS DE POTENCIA CIRCULANTE}

Este tipo de máquina (Figura 36), también es utilizada para evaluar el comportamiento de engranajes a la fatiga por flexión, montados en parejas y sometidos a una carga previamente establecida, se hacen girar engranados entre si, a velocidades preferiblemente inferiores a las $1000 \mathrm{rpm}$ para detener la máquina y evitar que se dañe, cuando ocurra la falla. Como en esta configuración los engranajes trabajan en rotación, el reto principal estriba en diseñar ensayos, donde no ocurran modos de fallas indeseados (picadura, agarramiento), lo cual requiere controlar las características constructivas de los engranajes y la propia metodología de ensayo, debiendo atenuarse los elementos que propicien la aparición de fallas no deseadas, antes de que se cumpla el número de ciclos requeridos. Esta constituye la principal desventaja que poseen estas máquinas, aunque los resultados alcanzados son más precisos que los obtenidos en los ensayos sobre diente único, especialmente si se eliminan o minimiza la influencia de otros modos de fallas sobre el ensayo [72]. 
Jorge L. Moya Rodríguez et al/ ITEGAM-JETIA Vol.02, № 06, pp.111-126. Junho, 2016.

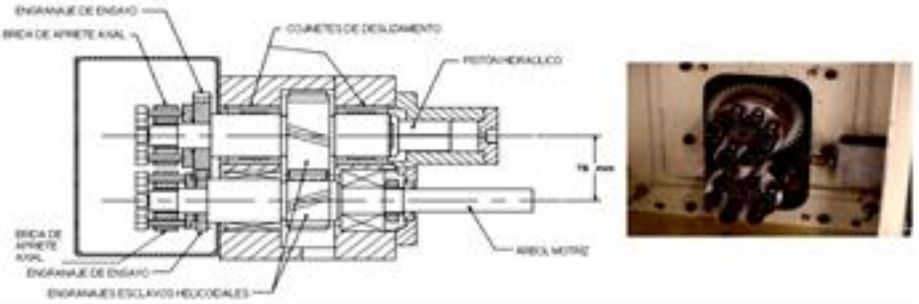

Figura 36 - Máquina de potencia circulante para el ensayo a la fatiga por flexión [76].

\section{MÉTODO DE ELEMENTOS FINITOS Y EXPERIMENTACIÓN, EN EL ANÁLISIS DE FALLAS}

En los últimos tiempos las transmisiones por engranaje acaparan la atención de muchos estudiosos y técnicos y como resultado de este interés han aparecido novedosos métodos de cálculo de esfuerzos en los dientes resueltos con la aplicación de modernas técnicas de análisis que han requerido de un intenso empleo de la computación y los procesamientos matemáticos. Dentro de estos procesamientos se destaca el Método de los Elementos Finitos (MEF), muy utilizado para el análisis de la resistencia en los dientes de los engranajes. El MEF en la actualidad se ha convertido en una herramienta potente y muy fiable y es frecuentemente utilizado como referencia para el análisis numérico, analítico y experimental de los resultados [7779].
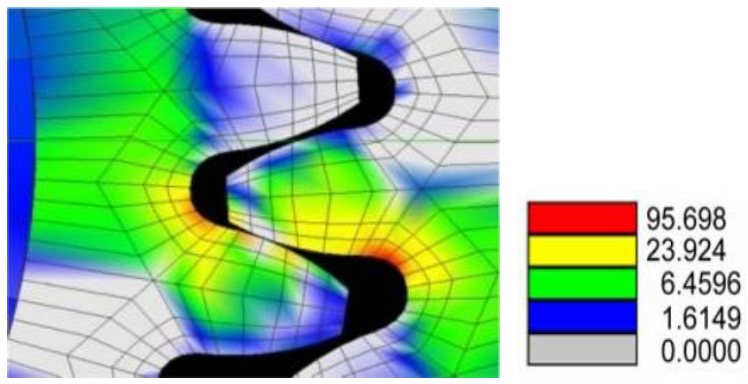

Figura 37 - Resultado de Análisis mediante el Método de Elementos Finitos mostrando la distribución de tensiones generadas entre dos engranajes [74].

Cuando se requieren efectuar determinaciones de estados complejos de tensión y deformación de componentes, el cálculo mediante simulación por ordenador desempeña un papel de gran importancia, debido fundamentalmente al ahorro económico (no se tiene que realizar un montaje físico ni utilizar componentes reales de los sujetos a estudiar), y al ahorro de tiempo. Lo dicho, parece situar a este método como único camino a la hora de plantear un ensayo.

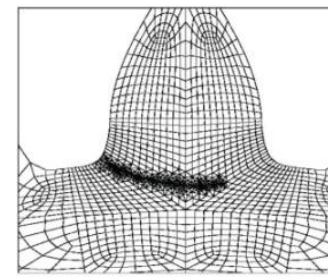

(a)

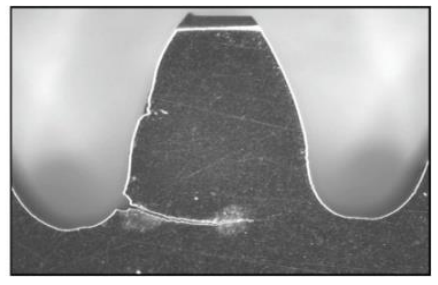

(b)
Figura 38 - (a) Trayectoria de la grieta simulada mediante MEF. (b) Propagación de la trayectoria de la grieta durante un ensayo [80].

Sin embargo no resulta así, la gran complejidad que se deriva de un fenómeno multifactorial como la fatiga, hacen que sea necesario recurrir a la experimentación para evaluar los aspectos que se escapan a las capacidades del cálculo. Por tanto, al estimar la vida a fatiga de un componente o conjunto de componentes, cálculo y experimentación son herramientas que lejos de ser sustitutiva una de la otra, deben ser complementarias en torno a un objetivo común [81].

A pesar del impresionante progreso hecho en los últimos años, relativos al análisis y simulación, la experimentación resulta aún esencial.

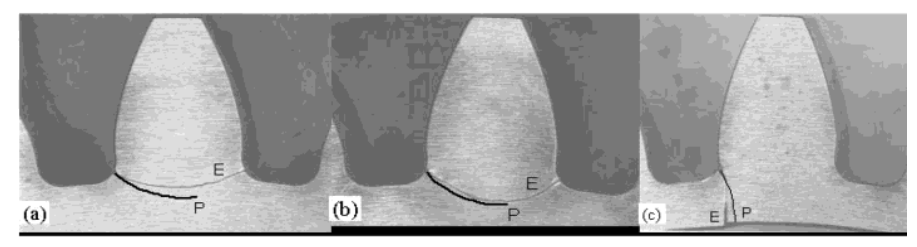

Figura 39 - Comparación de la predicción de propagación de la grieta $[\mathrm{P}]$ con los resultados experimentales [E] [82].

\section{CONCLUSIONES}

1. Los dos principales modos de fallas a los que se requiere comprobar una transmisión por engranajes, lo constituyen, las fallas a la fatiga por contacto superficial, y a la fatiga por flexión.

2. Las máquinas de ensayo del tipo engranaje, poseen una tendencia de empleo más favorable que las del tipo simulación, pues la correlación con el comportamiento real en servicio, ha sido mejor utilizando engranajes como especimenes de ensayo.

3. Existen varios dispositivos para aplicar el torque en las máquinas de ensayo de engranajes, resultando los sistemas hidráulicos, los que permiten una explotación más flexible y un mejor control y automatización del proceso. 
Jorge L. Moya Rodríguez et al/ ITEGAM-JETIA Vol.02, Nº6, pp.111-126. Junho, 2016.

4. Se requieren desarrollar dos máquinas para la verificación de la capacidad de carga de engranajes cilíndricos de dientes rectos, que permitan evaluar el comportamiento de estos elementos en relación con los dos criterios de diseño más difundidos y que sirven de base a diversas normas de comprobación para este tipo de engranajes, una máquina estaría destinada para realizar ensayos de resistencia a la fatiga por contacto y la otra para ensayos de resistencia a la fatiga por flexión.

5. La máquina a diseñar para la realización de ensayos a la fatiga por contacto, se recomienda sea del tipo engranaje, bajo el principio de potencia circulante, con sistema de cilindro rotatorio hidráulico para la aplicación del torque, construido en el interior de uno de los engranajes patrones, seleccionando como referencia para su diseño, la máquina desarrollada por el Centro de Investigación sobre Engranajes de la NASA, la cual además de poseer las múltiples ventajas que sobre este tipo de máquinas ya han sido relacionadas con anterioridad, poseen un notable aval refrendado por numerosas publicaciones que refieren sobre su exitoso uso durante un largo periodo de tiempo, en múltiples investigaciones relacionadas con engranajes.

6. Para los ensayos a la fatiga por flexión, se propone implementar un sistema del tipo pulsador, utilizando una máquina estándar para ensayos de fatiga por traccióncompresión, esta configuración posee un grupo de características ya citadas, que la hacen muy apropiada para estos ensayos, además de aportar una gran simplificación desde el punto de vista constructivo, pues solo se requiere desarrollar un aditamento especial para soportar el engranaje y garantizar la adecuada aplicación de la carga.

7. A pesar de los grandes progresos logrados en los últimos años en el análisis de engranajes mediante el método de elementos finitos, la experimentación continua desempeñando aun, un importante papel en este campo y sirve de complemento y validación, a los métodos computacionales y de procesamiento matemático.

\section{BIBLIOGRAFIA}

[1] Chen, Y., Yamamoto, A.; Omori, K. Improvement of Contact Fatigue Strength of Gears by Tooth surface Modification Processing, in 12th IFToMM World Congress. 2007: Besançon (France). p. 6.

[2] DeLange, G., Failure Analysis for Gearing. 2009. p. 12.

[3] Fernandes, P.J.L., Tooth Bending Fatigue Failures in Gears. 1996. p. 7.
[4] Fernandes, P.J.L., McDuling, C. Surface Contact Fatigue Failures in Gears. 1997, Pergamon. p. 9.

[5] Glodez, S., Winter, H.; Stüwe, H. P. A Fracture Mechanics Model for the Wear of Gear Flanks by Pitting 1997, Elsevier. p. 7.

[6] Loutridis, S., A local energy density methodology for monitoring the evolution of gear faults. 2004, Elsevier: Thessaloniki, Greece. p. 7 .

[7] Rexnor, Failure Analysis Gears-Shafts-Bearings-Seals. 1978, Rexnor Industries. p. 20.

[8] Dobrovolsky, V., Elementos de Máquinas. 1976, Moscú: Editorial MIR.

[9]ANSI/AGMA 1010-E95 Appearance of gear teethterminology of wear and failure. 2005. p. 48.

[10]Rivas, D. La Lubricación en la Gestión de Mantenimiento. 2008. 66.

[11]ISO 6336-2 Calculation of Load Capacity of Spur and Helical Gears, Part 2: Calculation of Surface Durability (Pitting). 2008.

[12] ISO 6336-3 Calculation of Load Capacity of Spur and Helical Gears, Part 3: Calculation of Tooth Bending Strength. 2008.

[13] Magalhaes, L., Seabra, J. Teeth Surface Failures in Austempered Ductile Iron (ADI) Gears. 2003, Universidad do Porto: Portugal.

[14] Mihailidis, A., Nerantzis, I. A New System for Testing Gears Under Variable Torque and Speed. 2009, Bentham Science Publishers Ltd.: Thessaloniki, Greece. p. 14.

[15]Moya, J.L., Goytisolo, R. A.; Hernández, A. E.; Machado, A. S. Simulación del Contacto en Transmisiones por Engranajes, in 8vo Congreso Iberoamericano de Ingeniería Mecánica. 2007: Cusco.

[16] Friction in to the gearing. Rev. of the ASME, 1888. 8 y 9.

[17] Efficiency of gear drives. Rev. Trans. of the ASME, 1918. 40: p. 107.

[18]Kenerson, W.H., Investigacion of efficiency of wor gearing for automoviles. Trans. of the ASME, 1912. 34: p. 919.

[19]Martin, H.M., The lubrication of gear teeth. Rev. Engineering, 1916. 102: p. 119.

[20] BeeK, O.W., Givens, I.; Smith, A. E. The mechanism of boundary lubrication. Rev Proc. Roy Soc, 1940: p. 9. 
Jorge L. Moya Rodríguez et al/ ITEGAM-JETIA Vol.02, № 06, pp.111-126. Junho, 2016.

[21] Mc Kee, S.A., Swindells, J. F.; White H. S.; Wayne, M. Gear Lubricant testing. Rev. Mech. Eng., 1949. 71: p. 67.

[22] Lane, T.B., Scuffing temperature of boundary lubricant films. Rev. Brit. Journal of App. Phys., 1951. 2: p. 35.

[23] Talliam, T.E., Lubricant film, rolling contact of rough surfaces. Rev. Trans. of the ASME, 1964. 7(2): p. 109.

[24]Crook, A.W., Simulated gear- tooth contacts: some experiments upon their lubrication and sub- surfaces deformation. Rev.Proc. Inst. Mech.Eng., 1957. 171: p. 187.

[25]Smith, F.W., Lubricant behaviour in concentrated contac systems the castor oil Steel system. Rev. Wear, 1959. 2: p. 250.

[26]Sibley, L.B., Orcutt, F.K. Elastohydrodynamics Lubrication of rolling contact surfaces. Rev Trans. of the ASME, 1961. 4: p. 234.

[27] Dawson, D., Lonfield, M. D. The lubrication of rolls finite with: An investigation of oil-film characteristics, in Rev. Instn. Mech. Eng. 1964.

[28] Archard, J.F., Kirk, M.T. Lubrication at point contacts. Rev. Proc. Roy. Soc., 1961. 261: p. 532.

[29] Oliver Fernández, D., Diseño de un banco de ensayo para árboles articulados. 2007.

[30] Lanahan, J.H., US3112643. 1963.

[31]Klinger, F., Prüfstände für Getriebe. Wellen, Kupplungen: Antriebstechnik, in 11. 1972. p. 332-336.

[32] Langenbeck, K., Getriebeprüfstände für Forschung und Entwicklung, in 115, VDI-Z, Editor. 1971. p. 115-121.

[33] Basedow, G., Prüfstände mit verspannten Getrieben. Antriebstechnik, 22, Editor. 1983. p. 32-38.

[34] Gruschka, B., Herrmann, B. DE3100848. 1982.

[35] Musser, C.W., US2929266. 1960.

[36] Schröder, G., Hüser, H.; Krug, H. DE4325403. 1995.

[37] Brüggemann, D., Hüser, H.; Fischer, W. G. DE10119148. 2002.

[38] Harald, A., Löhr, H. DE2908860. 1980.

[39] Yano, M., Yoshihara, Y. US5113704. 1992.

[40] Bader, A., DE102006014237. 2006.

[41] Collins, L.J., US2371607. 1945.
[42] Hennings, G., Ein Zahnradverspannungsprüfstand mit einstellbarem Belastungsprogramm. Maschinenbautechnik, (5), Editor. 1970. p. 259-262.

[43] Schneider, H., Bartsch, K.; Schucht, K. DD249392. 1987.

[44] Shipley, E.E., US2935869. 1960.

[45] Kugler, A., Roth, A. DE3424923. 1986.

[46] Mihailidis, A., GR20090100039. 2009.

[47] Wolfrom, U., Der Wirkungsgrad von Planetengetrieben. Werkstatttechnik. 1912. p. 615-617.

[48] AGMA 925-A03 Effect of Lubrication on Gear Surface Distress. 2005. p. 58.

[49] Townsend, D.P., Chevalier, J. L.; Zaretsky, E. V. Pitting fatigue characteristics of AISI M-50 and super nitralloy spur gears. 1973, NASA: Washington, D. C. p. 24.

[50]Winter, H., Zahnraduntersuchungen im Verspannungsprüfstand. Das Industrieblatt. 1955. p. 433437.

[51] Hoehn, B.R., Oster, P.; Tobie, T.; Michaelis, K. Test Methods for Gear Lubricants. 2008.

[52] DIN 51354: Standard test method for lubricants using the FZG gear test-rig. 1990.

[53]CEC L-07-A-95: Load-carrying capacity for transmission lubricants. 1990.

[54]IP 334: Determination of load-carrying capacity of lubricants-FZG gear machine method. 1993.

[55] ASTM D5182: Standard test method for evaluating the scuffing load capacity of oils (FZG visual method). 1997.

[56] ISO 14635: FZG test method A/8.3/90 for relative scuffing loadcarrying capacity of oils. 2000.

[57] ASTM D4998: Standard test method for evaluating the wear characteristics of tractor hydraulic fluids. 1995.

[58] GFC T 014 T 85. Ecotrans method - Assessment of the ability of lubricants to reduce friction losses in transmission. 1985.

[59] FVA 345/1: Standard test method for determining the lubricant's effect on friction coefficients using the FZG geartest rig. 2002.

[60]FVA 54/I-IV: Standard test method for determining the lubricant's effect on the formation of micro-pitting - Test method GF-C/8.3/90. 1993. 
[61] FVA 2/IV: Standard test method for determining the lubricant's effect on the life cycle of pitting in load stage test: PT-C/9/90, PTC/ 10/90. 1997.

[62] FVA 371: Load collective test: PT-C/LLS/90, PTC/HLS/90 development of a praxis relevant pitting test. 2006.

[63]Langenbeck, K., Die Verschleiß- und Fressgrenzlast der Hypoidgetriebe., in VDI-Z. 1967. p. 213-225.

[64]Langenbeck, K., $\quad$ Schraubenräder zur Leistungsübertragung, VDI-Z, Editor. 1969. p. 257-260.

[65]Podgornik, B., Vizintin, J. Sliding and Pitting Wear Resistance of Plasma and Pulse Plasma Nitrided Steel. 2001, 2001 IoM Communications $L$ td.

[66] Morris, J., Goldsworthy, J.; Scott, J.; Sauter, C. Development of a Gear and Spline Lubricant Tester. 1954, Western Gear Work: Ohio. p. 91.

[67]IP 166/65: Load-carrying capacity for oils. IAE gear machine. 1965 .

[68]Zaskal'ko, P.P., Kuznetsov, E. G.; Krysin, V. D.; Chechetkin, V. V. Evaluation of antiscuff properties of transmission oils in IAE tester by qualification test procedure. Khimiya i Tekhnologiya Topliv i Masel, 1976(7): p. 54-57.

[69] Zaskal'ko, P.P., Krysin, V. D.; Zabelin, A. V. Evaluation of Load-Carrying Capacity of Oils in IAE Gear Machine with Smaller Oil. Volume. 1983, Plenum Publishing Corporation.

[70] ASTM D1947-83: Standard test method for load carrying capacity of petroleum oils and synthetic fluid gear lubricants. 1983.

[71] Ryder, E.A., A gear and lubricant tester - Measures tooth strength or surface effects, A. Bull, Editor. 1947. p. 6973.

[72]McPherson, D.R., Rao, S. B. Experimental Characterization of Bending Fatigue Strength in Gear Tooth, in Geartechnology. 2003. p. 8.

[73] Krantz, T., Tufts, B. Pitting and Bending Fatigue Evaluations of a New Case-Carburized Gear Steel. 2007, NASA: Cleveland. p. 14.

[74] Handschuh, R.F., Krantz, T. L. Investigation of LowCycle Bending Fatigue of AISI 9310 Steel Spur Gears. 2007, NASA: Ohio. p. 14.

[75] Wulpi, D.J., Shot Peened Gears as Evaluated by Single Tooth Fatigue Test. 1956.
[76] Nigarura, S., Parameswaran, R.; Trasorras, J. R. Bending Fatigue of Surface Densified Gears: Effect of Root Densification Depth and Tooth Loading Mode on Fatigue Life. 2006. p. 14

[77] Litvin, F.L., Chen, J.S.; Lu, J. Application of Finite Elements Method for Determination of Load Share, Real Contact Ratio, Precision of Motion, and Stress Analysis. Journal of Mechanical Design, 1996. 118: p. 561-567.

[78]Muthukumar, R., Rhagavan, M. R. Estimation of Gear Tooth Deflection by the Finite Elements Method. Mechanism \& Machine Theory, 1987. 22: p. 177-181.

[79] Wilcox, L., Coleman, W. Application of Finite Elements to the Analysis of Gear Tooth Stresses. ASME Journal of Engineering for Industry, 1973. 95: p. 1139-1148.

[80] Li, C.J., Choi, S. Spur Gear Root Fatigue Crack Prognosis via Crack Diagnosis and Fracture Mechanics. 1998. p. 10.

[81] Wei, Z., Stresses and Deformations in Involute Spur Gears by Finite Element Method. 2004.

[82] Mosher, M., Huff, E. M., Modelling of Vibration Measurements for Gear Fault and Damage Detection on Aircraft. 2004. 